\title{
Outreaching particle physics to Latin America: CEVALE2VE and the use of ATLAS open data
}

\author{
Reina Camacho Toro*† \\ University of Chicago (US) \\ E-mail: reina.camacho@cern.ch
}

Particle physics outreach can play a key role in promoting scientific culture and in the modernisation of university education in Latin America. In this context, the CEVALE2VE virtual community (Centro de Altos Estudios de Altas Energías in Spanish) builds collaborative networks with and between Latin American institutions and motivates physics undergraduate and master students to consider a career in scientific research by introducing them to cutting-edge research and tools used in High Energy Physics (HEP). The ATLAS open data project has been fundamental to achieve these goals as one of our main educational tools. As part of its commitment to open access and public engagement the ATLAS experiment has made available a large dataset to the public for the first time in 2016 and CEVALE2VE has been using this dataset for HEP virtual courses and Physics Without Frontiers roadshows. In this contribution we will present the different activities hosted by CEVALE2VE and discuss the importance of using open data and open software to reach strong young students lacking exposure to particle physics research.

The European Physical Society Conference on High Energy Physics

5-12 July, 2017

Venice

*Speaker.

$\dagger$ on behalf of the ATLAS collaboration and the CEVALE2VE community 


\section{Introducing the CEVALE2VE community}

CEVALE2VE (Centro de Altos Estudios de Altas Energías in Spanish) [1] is a virtual research and learning community created in 2014 with the goal of promoting the scientific dissemination, education and research in the field of High Energy Physics (HEP) in Venezuela and Colombia. Although geographically scattered in different institutions of Europe and North America, CEVALE2VE integrates a group of 12 Venezuelan and Colombian researchers working in international collaborations: HEP experiments and data science, all with particle physics background (50\% of the group are members of the ATLAS Collaboration [2]). The four main objectives of this community are:

- Education and outreach: stimulate physics student's interest in HEP research.

- Awareness of opportunities: educate and invigorate the students with further study opportunities in physics and possible career paths in research.

- Create networks: in both directions!

- Modernization of the education and training: through the use of a web platform with e-learning tools.

- Policy making and diplomacy: e.g working towards formalising the involvement of Venezuela institutions in HEP experiments.

\section{CEVALE2VE activities}

CEVALE2VE has three development axes: education, outreach and policy making. In this document emphasis will be given to the first two axes, the last one is related with communicating our projects and results to the public, diplomatic members and other governmental institutions, as well as building links between academy and industry.

\subsection{Education related activities}

The main activity is the implementation of a 60 hours virtual/online course "Introduction to Particle Physics" [3] developed for master and PhD students with little or no HEP background/experience. The course goal is to provide students with an overview of the concepts and methods used in contemporary particle physics research. The program is divided in five modules: introduction to the Standard Model (SM) and physics Beyond the SM; accelerator principles and particle detector principles; introduction to statistics, data analysis and computer tools based in ROOT [4] and a series of seminars by invited speakers working in relevant areas of physics. The classes are in Spanish and the written materials are in English.

The course was officially included in the postgraduate course portfolios of five public universities and research institutions, thanks to the support of local professors, namely the Universidad Central de Venezuela (UCV), the Universidad Simón Bolívar (USB), the Universidad de Los Andes (ULA), the Universidad Industrial de Santander (UIS) and the Universidad Antonio Narino (UAN) and informally followed by several students from the Universidad de Carabobo (UC), the Instituto Venezolano de Investigaciones Científicas (IVIC) and the Universidad del Zulia (LUZ). Since 2014 


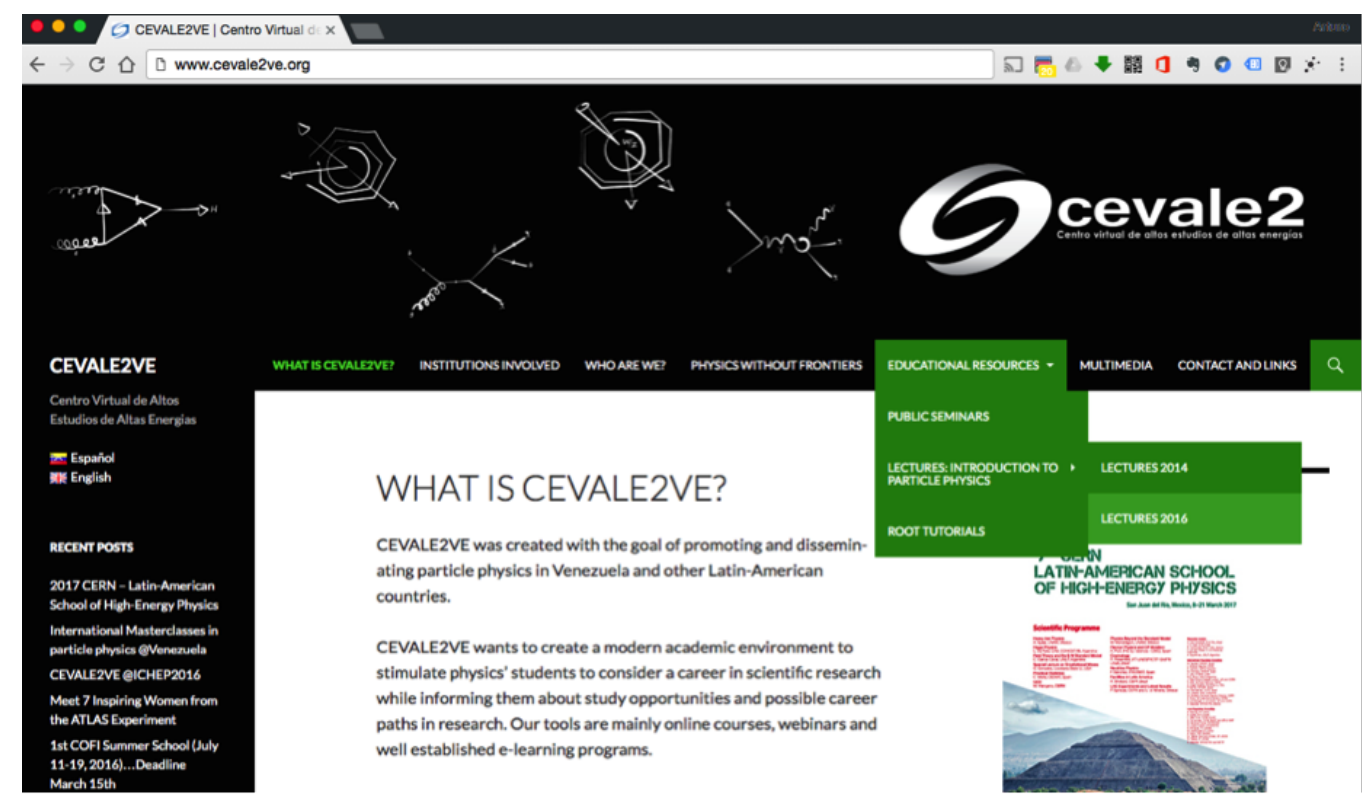

Figure 1: The CEVALE2VE web platform [1] which contains a web-based repository of all of the online particle physics courses and seminars.

the course has already 3 editions with more than 40 students who attended classes and 20 accredited students. Another important activity for the community is the supervision of undergraduates and master students theses.

The use of e-learning tools has been fundamental for CEVALE2VE. The classes were possible using Google Hangouts [5] and transmitted and recorded in YouTube [6] (see Figure 2). Currently there are more than 100 hours of online audio-visual content available for the public. The Moodle online platform [7] at UCV and the CEVALE2VE web page [1] was used for off-line discussions, homework submission and general announcements (see Figure 1). In particular the release of the ATLAS open data in 2016 allowed us to improve the data analysis module in the course.

\subsection{Outreach related activities}

The outreach events of CEVALE2VE are meant to be a way to discuss the benefits of the scientific activities taking place in international organizations like CERN and others around the world. At the same time to let the nationals of Latin American countries know how they can contribute to this global scientific enterprise. This list summarises the group's outreach related activities:

- Public seminars in Spanish presented by CEVALE2VE members or invited physicists working in non-HEP areas like data analysis and economics.

- Venezuelan institutions joined the International Particle Physics Outreach Group (IPPOG) Masterclasses [8] program in 2016 for the first time.

- ATLAS Virtual Visits [9].

- Partnership with the ATLAS collaboration outreach group and the Abdus Salam International Centre for Theoretical Physics (ICTP)'s Physics Without Frontiers (PWF) [10] program since 


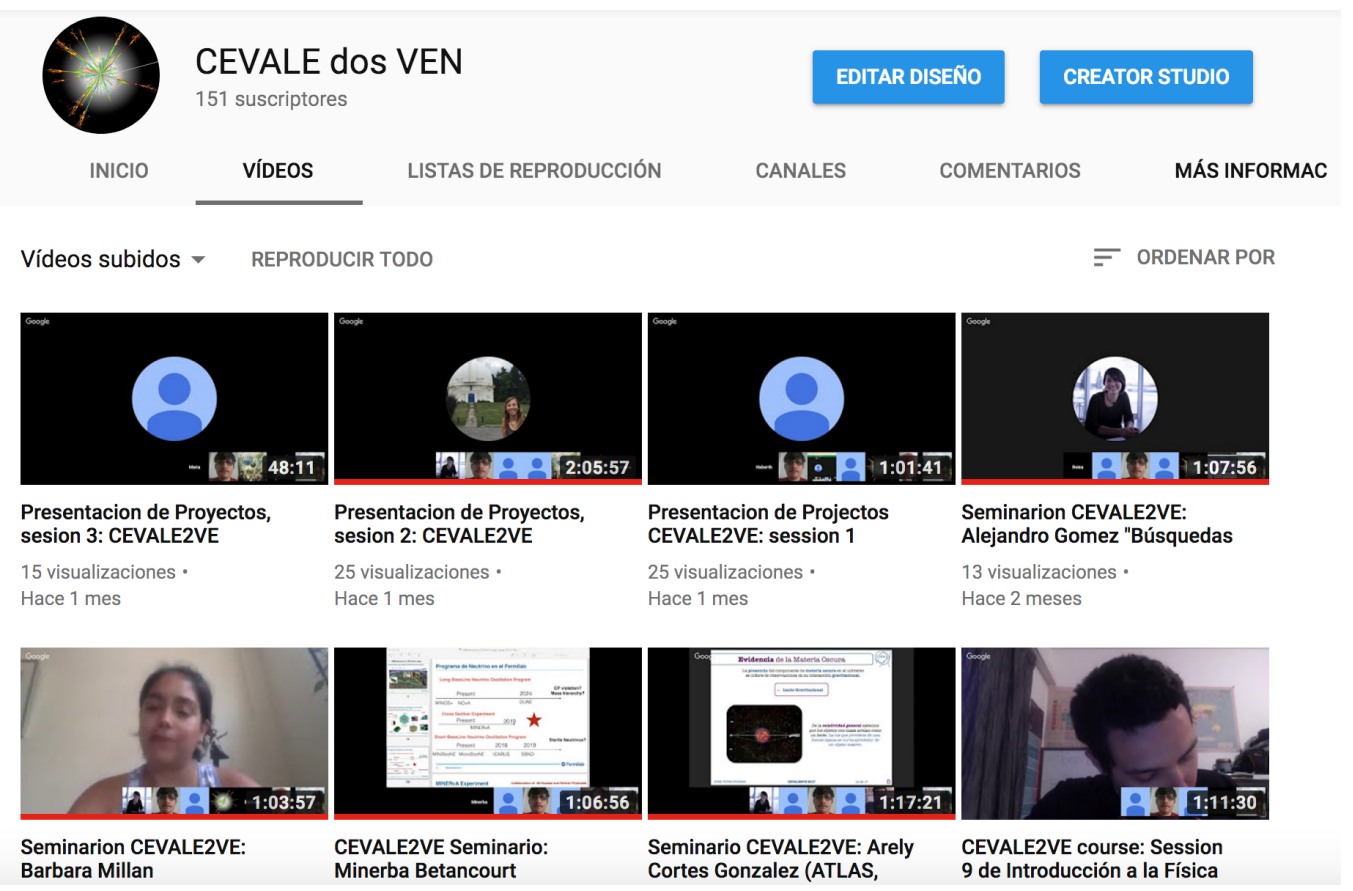

Figure 2: The CEVALE2VE YouTube channel [6], which contains more than 100 hours of online audiovisual content and 9000 views and dozens of comments in the tutorials.

2016. Five Colombian and Venezuelan universities were visited alongside a substantial outreach program to inspire and engage physics students with cutting-edge research using data from the ATLAS experiment at CERN, and motivate them to continue their studies in a period of 2 weeks in October 2016.

\section{ATLAS Open Data and CEVALE2VE}

The release of the ATLAS open data [11] marked the world's first open release of $8 \mathrm{TeV}$ data, gathered from the Large Hadron Collider in 2012. A hundred trillion proton collisions are contained in this dataset, which corresponds to an integrated luminosity of $1 \mathrm{fb}^{-1}$. Event selection was carried out, reducing the size and information content of the standard data format used in ATLAS, enabling faster event processing. The resulting dataset can be used for various analyses. The ATLAS Open Data is ideal for university-level students and educators. To help users analyse these data, ATLAS has also launched a comprehensive educational platform, as well as tools and software of varying technical difficulty to analyse the data.

Currently there are more than 15 projects using the ATLAS Open Data around the world (see Figure 3). A number of university lecturers have used the educational platform directly with undergraduates or developed courses around the platform. University summer students and high school students visiting CERN have also used the educational platform to learn about ATLAS and particle physics.

CEVALE2VE ATLAS members helped test the ATLAS Open Data in its earlier versions, before its release. We used the official released ATLAS Open Data in 2016 during the PWF program 

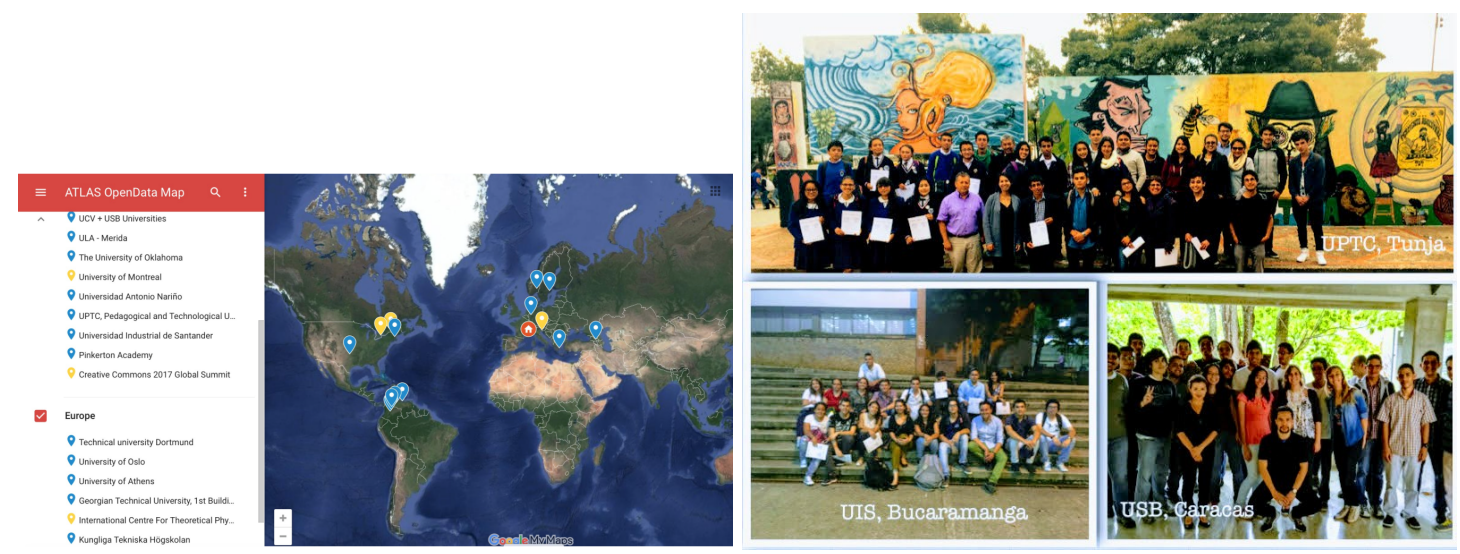

Figure 3: Google maps image showing location/users of the ATLAS Open Data educational platform [11] (left) and images from the Colombian and Venezuelan undergraduate and masters students who followed a one day master class in October $2016[8,9,10]$

in Colombia and Venezuela. We included it in the data analysis module of the third edition of the online course "Introduction to Particle Physics" that took place between March and July 2017. It was motivating for the students to have access to real data, since most of the participating institutions are not involved in LHC experiments. Also, the first master thesis using the ATLAS Open Data was finished this year. The student was part of our first session of the course in 2014-2015 at the UCV university and worked on the evaluation of Dark Matter production in association with quarks or electroweak bosons in colliders like the LHC. The fact that the final dataset fits onto a typical USB key (under $11 \mathrm{~GB}$ ), easing storage issues, is important for us because the IT infrastructure in some places in Latin America can be fragile and restrictive.

\section{Challenges and perspectives}

CEVALE2VE have implemented several HEP educational and outreach projects in Venezuela and Colombia in the last few years. The ATLAS Open Data project has been a key element in those activities. The main challenges we have faced are related to the fragile and restrictive IT infrastructure in some of the participating institutions and the fact that the student computer skills did not match the initial demands. The outcomes are invaluable: motivating students, some of whom follow a career in HEP now ( 8 of them have participated in HEP international schools, 4 are taking a masters in HEP and one is following $\mathrm{PhD}$ studies). We seek to extend our partnerships with other institutions and individual collaborators in order to reach more students and more regions and to be able to provide more opportunities to the students (internships on site, theses projects, etc).

\section{References}

[1] CEVALE2VE website - http://www.cevale2ve.org/ (Cons. 01/09/2017).

[2] ATLAS Collaboration, The ATLAS Experiment at CERN Large Hadron Collider, JINST 3 (2008) 03.

[3] CEVALE2VE virtual course http://www.cevale2ve.org/en/lectures/particle-physics-introductory-course/ (Cons. 01/09/2017). 
[4] R. Brun and F. Rademakers, ROOT - An Object Oriented Data Analysis Framework, Nucl. Inst. \& Meth. in Phys. Res. A 389 (1996) 81-86.

[5] Google Hangouts - https://hangouts.google.com (Cons. 01/09/2017).

[6] CEVALE2VE YouTube video hosting service - www.youtube.com/cevale2ve (Cons. 01/09/2017).

[7] Campus Virtual de la UCV - http://campusvirtual.ucv.ve/ (Cons. 01/09/2017).

[8] IPPOG Masterclasses program - http://physicsmasterclasses.org (Cons. 01/09/2017).

[9] ATLAS Virtual Visits - http://atlasvirtualvisit.web.cern.ch/content/latin-america (Cons. 01/09/2017).

[10] ICTP Physics Without Frontiers program - https://www.ictp.it/physics-without-frontiers.aspx (Cons. 01/09/2017).

[11] The ATLAS Open Data portal - http://opendata.atlas.cern (Cons. 01/09/2017). 